\title{
8 \\ http://dx.doi.org/10.21103/Article11(2)_RA1 \\ Twindemic of Coronavirus Disease (COVID-19) and Cardiometabolic Diseases
}

INTERNATIONAL JOURNAL OF BIOMEDICINE

\author{
Gundu H. R. Rao, Emeritus Professor \\ Laboratory Medicine and Pathology \\ Director of Thrombosis Research, Lillehei Heart Institute \\ University of Minnesota, Minneapolis, USA
}

\begin{abstract}
Metabolic diseases, like hypertension, excess weight, obesity, type 2 diabetes, and vascular diseases, have rapidly increased to epidemic proportions worldwide. Metabolic risks (such as oxidative stress, chronic inflammation, insulin resistance, altered glucose and lipid metabolism, changes in hemodynamics, endothelial dysfunction, and subclinical atherosclerosis) contribute significantly to the progress of vascular disease and drive it eventually to acute vascular events like heart attacks and stroke. Although this situation has been noticed and discussed extensively by the global public health experts, and professional societies, the unprecedented SARS$\mathrm{CoV}-2$ pandemic has demonstrated for the first time the interdependency or syndemic nature of metabolic diseases and a pathogenic virus that takes advantage of the compromised metabolic function in these diseases. The most common clinical symptoms reported are fever, cough, fatigue, shortness of breath, dyspnea, chest pain, sore throat, and sputum production. The main mode of transmission is through respiratory particles containing viral virions. Both asymptomatic and symptomatic patients seem to be infectious. The spike (S) protein of SARS-CoV-2 seems to have a 10- to 20-fold higher affinity to the human ACE2 receptor than that of SARS-CoV. Since these receptors are highly expressed on a variety of cells, including vascular endothelial cells and adipose tissue, individuals with compromised function of these tissues are more vulnerable to greater infection, replication, and severity with COVID-19. In most cases, the severity of the coronavirus disease is associated with pre-existing comorbidities, which include metabolic diseases such as hypertension, obesity, diabetes, and vascular diseases. Those with such diseases, or with elevated risk factors for such diseases, will have a compromised endothelium, favoring endothelial dysfunction. The infection of the endothelium by SARS-CoV-2 and resulting endothelialitis seems to add to this problem by further damaging the endothelium, causing dysfunction, disruption of vascular integrity, and endothelial cell death. These events lead to the exposure of the thrombogenic basement membrane and result in the activation of the thrombotic and clotting cascade. Because of these observations, critical care clinicians recommend aggressive anti-thrombotic and thrombolytic therapies in the management of acute COVID-19 cases. In the absence of a cure for coronavirus disease, sensible medicine proposes the following: primary prevention by following the best public health practices, such as social distancing, use of face coverings, and quarantine of COVID-positive individuals; and a gentler, moderate, and humble view and application of available treatment options and their effectiveness in patients with COVID-19. The FDA has created a special emergency program for possible coronavirus therapies, the Coronavirus Treatment Acceleration Program (CTAP). Currently, there are 590 drug development programs in planning stages, 390 trials in review, and five authorized for emergency use. None are approved for use in COVID-19 management. Currently, there are at least 51 studies listed in the COVID-19 vaccine tracker of the Regulatory Affairs Professional Society (RAPS) site. At the time of this writing, vaccines from Pfizer-BioNTech, Moderna, Oxford-AstraZeneca, and Johnson\&Johnson have emergency use authorization in the US. (International Journal of Biomedicine. 2021;11(2):111-122.)
\end{abstract}

Key Words: SARS-CoV-2 $\bullet$ COVID-19 • vaccine $\bullet$ metabolic diseases

For citation: Rao GHR. Twindemic of Coronavirus Disease (COVID-19) and Cardiometabolic Diseases. International Journal of Biomedicine. 2021;11(2):111-122. doi:10.21103/Article11(2)_RA1

\section{Introduction}

A novel coronavirus SARS-CoV-2, which escaped from Wuhan, China, in early January of 2019, has caused

Contact Information: Emeritus Professor Gundu H.R. Rao, Laboratory Medicine and Pathology, Director, Thrombosis Research, Lillehei Heart Institute, University of Minnesota. E-mail: gundurao9@gmail.com unprecedented healthcare and economic crisis worldwide (1-9) $^{(-9)}$ This $\$ 16$ trillion killer virus has caused havoc in all countries, and now after almost a year, has mutated into much more sophisticated 'new novel' virus variants, with a very high rate of transmission. ${ }^{(10,11)}$ The spreading rate of the British and South African variants seems to be greater than $70 \%$, compared to the normal SARS-CoV-2 virus. Variations in the UK variant seem to occur at the level of N501Y of the spike protein and involve 
23 separate mutations of the spike. ${ }^{(11)}$ Globally, according to Johns Hopkins University in Baltimore, Maryland, USA, over 112 million (mil) individuals are COVID-19 positive with 2.4 million deaths. The USA (28 mil), India (11.0 mil) Brazil (10.3 mil), Russia (4.16 mil), and the UK (4.15 mil) are competing for the top five ranks in terms of the number individuals COVIDpositive. In COVID-related deaths, the USA, Brazil, Mexico, India, UK, and Italy are the top-ranking countries. In early April 2019, when I published my first article on this topic, the rankings were different: there were 2.9 million infected individuals worldwide with 203,670 deaths. Confirmed cases ranked as follows: USA $(941,628)$, Spain $(223,759)$, Italy $(195,351)$, France $(161,665)$, Germany $(157,026)$, United Kingdom $(149,569)$, Turkey $(107,773)$, Iran $(90,481)$, China $(83,909)$, Russia $(80,949)$, and Brazil $(59,479)$. India with the second largest population and second-highest number of diabetics, with relatively poor healthcare infrastructure (1.2\% GDP VS $17 \%$ in the USA), ranked 16th with 26,917 cases. Malaysia and Bangladesh were way far below in their rankings. If one were to rank these countries based on case fatality rates (CFRs), then the ranking was as follows; Mexico (87\%), Italy (3.5\%), Hungary (3.1\%), Indonesia (2.9\%), South Africa (2.7\%). India with $1.4 \%$ CFR, ranked number 19.

COVID medicine and the public health response to this pandemic is a fast-changing field, with many controversies. The authors of CovidReference.com have been making every effort to provide updates on this topic with the mini-series; the most recent is the sixth edition of this mini-book. ${ }^{\left({ }^{6}\right)}$ According to the authors, in December of 2019, several patients from Wuhan developed pneumonia and respiratory failure. In early January 2020, a beta-coronavirus, later named SARS-CoV2, was isolated from the bronchoalveolar lavage of patients. From the first identification of COVID-19 in Wuhan, to the time of the first publication of this reference book (January 13, 2020), SARS-CoV-2 had spread to every corner of the world. According to Nobel laureate Joshua Lederberg, the success of the "wonder drugs" of the 1950s led to the belief that the "war on microbes" had been won, but the emergence of new infectious agents such as HIV and Ebola has shattered that illusion. In a speech in 1989, Lederberg warned against complacency in the age-old contest between mankind and microbes-viruses and bacteria. He laid out why man's "only real competitors remain viruses"; he further explained how all terrestrial life "is a dense web of mutualism and genetic interactions"; there are abundant sources of genetic variation "for viruses to learn new tricks (as proved by SARS-CoV-2), not necessarily confined to what happens routinely or even frequently." Now we have the proof of his prophecy, SARS-CoV-2, a novel and intelligent virus, if one may call it that, which has acquired multiple reading frames to delete unwanted mutations. One feels as if it has used artificial intelligence, pattern recognition, and machine learning techniques to become a highly contagious and most potent killer virus. The novel virus has improved over all the other members of this family, by acquiring the biochemical and structural features essential for rapid transmission, transfection, and replication by avoiding and deleting deleterious mutations and keeping favorable ones.

A recent comment published in the Lancet says that
COVID-19 is not a pandemic, it is a syndemic. ${ }^{(12)}$ Syndemics involve the clustering of two or more diseases within a population; the biological, social, and psychological interaction of those diseases; and the large-scale social forces that precipitate disease clustering in the first place. ${ }^{(13-15)}$ According to the experts, a syndemic approach reveals biological and social interactions that are important for prognosis, treatment, and health policy. Therefore, limiting the harm caused by SARSCoV-2 will demand far greater attention to non-communicable diseases (NCDs), and socioeconomic inequality than has hitherto been admitted. Disparities that exist in the rate of infection and severity of this disease in the African American community and other minority communities substantiate this observation by the experts. For argument's sake, COVID-19, for instance, is a pandemic over another pandemic — cardiometabolic diseases. (16-18) If we were to stretch our imagination further, we would have to include all the metabolic disease risk factors also as coexisting conditions. Addressing COVID-19 management, therefore, means, addressing all the metabolic risk factors as well as metabolic diseases such as hypertension, excess weight, endothelial dysfunction, inflammation, obesity, diabetes, vascular diseases, and chronic respiratory diseases. The SARS$\mathrm{CoV}-2$ pandemic is a nightmare for workers in public health and critical care. The effect of this pandemic will stay with us for a long time to come. In this overview, we will discuss coronavirus disease and its syndemic nature on cardiometabolic risks, and metabolic and vascular diseases.

\section{SARS-CoV-2 Biochemistry}

The first human coronavirus was first discovered and characterized by June Almeida a virologist in St Thomas Hospital in London in 1964. Since that time, five new human coronaviruses have been identified, including the severe acute respiratory syndrome virus, which caused significant mortality. Coronaviruses are single-stranded RNA virus genomes. The virion consists of genomic RNA embedded in phospholipid double layers and coated with two different types of nucleocapsid proteins $(\mathrm{N})$. The membrane (M) protein (a type of transmembrane glycoprotein), the envelope (E) protein, and the surface (S) protein. Beta coronaviruses have a shorter spike-like surface protein called hemagglutinin esterase. The lipid bilayer envelope of the virus protects the membrane proteins and the nucleocapsid proteins when the virus survives outside the host.

Coronaviruses have positive-sense RNA genomes, consisting of six conserved proteins. The conserved proteins are the polyproteins ppla and pplb, which encompass multiple protein domains involved in various aspects of coronavirus genome replication. The size of this virus is between 60 nanometers $(\mathrm{nm})$ to a maximum of $140 \mathrm{~nm}$. Respiratory droplets are typically 5-10 micrometers, and each droplet may contain 250 virions, which means just normal talking can generate more than 750,000 virions. In the high-case fatality rate (CFR) strain, the nucleocapsid protein and the spike protein are significantly enriched. The $\mathrm{N}$ protein is multifunctional, contributes to viral transcription efficiency and pathogenicity. The SARS-CoV-2 spike proteins bind to the ACE2 host receptor with a 10 to 20 - 
fold affinity compared to SARS-CoV and contain a polybasic furin cleavage site, resulting in a unique insert to SARS-CoV-2 that enhances infectivity. Cleavage of $\mathrm{S}$ protein generates a polybasic Arg-Arg-Ala-Arg C-terminal sequence on S1 and S2. Furthermore, their analysis revealed a four-amino acid insertion in the long connecting region between the fusion peptide of the spike protein, in all high-CFR viruses, but not in low-CFR ones. Yet another difference they noticed was increased positive charge of the amino acids, comprising the nuclear localization signals (NLSs), a known marker of NLS strength. ${ }^{(19)}$ Recent findings suggest the interaction of yet another receptor, called neuropilin-1(NRP1), and neuropilin 2 (NRP2) that facilitate the entry of this virus into cells. Key cell entry mechanism includes higher ACE2 (hACE2) binding affinity of the spike to the receptor-binding domain, reduced dependence on target cell proteases for entry, due to pre-activation by convertase furin. ${ }^{(20)}$

\section{Transmission}

The spike protein is a type 1 transmembrane protein, comprising 1255 amino acids and seems to be the key to the host cell interactions. The virus has undergone significant mutations as it has evolved worldwide. However, the $\mathrm{S}$ protein seems to be the key determinant of evolution, transmission, and virulence of SARS-CoV-2. Coronavirus entry into host cells is mediated by the transmembrane spike (S) glycoprotein that forms homotrimers protruding from the viral surface. ${ }^{(21)}$ This protein comprises two functional subunits, responsible for binding to the host cell receptor (S1subunit), and fusion of the viral and cellular membranes (S2 subunit). For all viruses of this group, the $\mathrm{S}$ unit is cleaved by host proteases at the $\mathrm{S} 2$ site of the fusion peptide. Because of this mode of transmission, coronavirus entry into the host cell is a complex process, that requires both receptor binding and proteolytic processing of the $\mathrm{S}$ protein, to promote virus-cell fusion. SARS-CoV-2 entry into a cell involves the interaction of its spike protein with the cell's membrane-bound angiotensin-converting enzyme 2 (ACE2), which is cleaved by the transmembrane protease serine 2 (TMPRSS2), suggesting that co-expression of both genes is required for infection. ${ }^{(22)}$

The step between the SARS-CoV-2 spike glycoprotein and the ACE2 receptor seems to be the most critical point for the entry of the virus into the host cells. The high affinity of the $\mathrm{S}$ protein to the human ACE2 receptor seems to facilitate the spread of this virus in human populations. According to experts, four important enzymes are essential for the pathogenesis: the $\mathrm{S}$ protein that facilitates virus entry through the ACE2 to the host cell surface receptor, the major protease of CoV3Clpro, the papain-like protease (PLpro) involved in the assembly of new viruses, and RNA-dependent polymerase ( $\mathrm{RdPr}$ ) that facilitate CoV RNA genome replication. The processing and activation of coronavirus $\mathrm{S}$ protein are critical for the infectivity of the virus. The proprotein convertase family (PC) is composed of nine serine-secreting proteases and is widely involved in regulating various biological processes in normal and disease states. Therefore, the PC family, especially Furin, can be considered the key player that mediates the maturation of the $\mathrm{S}$ protein processing and recognition of membrane proteins. According to the experts, Furin can be considered a critical molecule that makes SARS-COV-2 cause adverse cardiovascular events through the ACE receptor. ${ }^{(23)}$

\section{Clinical Manifestations}

Researchers from the department of statistics, University of Dhaka, published a Meta-Analysis on clinical manifestations and comorbidities of coronavirus infection. ${ }^{(24)}$ Of the total of 33 eligible studies, including 7673 infected patients, the most prevalent clinical symptoms were fever $(84.49 \%)$, cough (56.39\%), fatigue (33.65\%), dyspnea (22.34\%), sputum production $(22.34 \%)$, and myalgia (16.26\%). Other symptoms included shortness of breath, diarrhea, headache, chest pain, vomiting, sore throat, and poor appetite, loss of smell and taste, and chills. The most prevalent comorbidity was hypertension $(20 \%)$, cardiovascular disease $(11.9 \%)$, and diabetes $(9.8 \%)$. Other less know comorbidities include excess weight, obesity, chronic kidney disease, chronic liver disease, chronic pulmonary disease, and cerebrovascular disease. ${ }^{(25-29)}$ These viruses enter the nasal epithelial cells, using the surface spike (S) protein to bind ACE2, which serves as receptors for SARS-CoV-2, on the bronchial epithelial cells and type II pneumocytes. Researchers have analyzed the ACE2 RNA expression profile at single-cell resolution. High ACE2 (hACE2) expression has been identified in type II alveolar cells of the lung, esophagus, enterocytes of the ileum and colon, cholangiocytes, myocardial cells, kidney proximal tubule cells, bladder urothelial cells, fat cells, and vascular endothelial cells. ${ }^{(20-22)}$

Following infection and viral replication, downregulation of ACE2 enzyme occurs, resulting in dysfunction of the angiotensin system, resulting in hypokalemia, vasoconstriction, and development of acute respiratory distress syndrome (ARDS). Endothelium, comprising a monolayer of endothelial cells is the largest organ of the body, covering a large surface area, and reaching out to every tissue and organ. Based on emerging evidence from patients with COVID-19, experts postulate that endothelial cells are essential contributors to the initiation and propagation of severity of COVID-19. Because of these observations, one can further speculate that the injury to the endothelium could introduce a cascade of events, leading to platelet activation, thrombin generation, and promotion of both thrombotic and thrombolytic events (Figure 1).

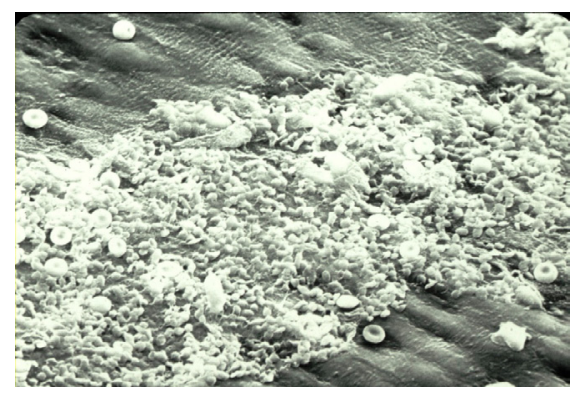

Fig. 1. Platelet interaction with injured endothelium. (Courtesy: Prof. James G. White).

To distinguish the term 'vascular disease' from the vascular damage and pathology observed in the severely ill 
Covid-19 patients, we refer to this condition as a disease of the blood vessels. ${ }^{(29)}$ In most cases, the severity of the coronavirus disease is associated with pre-existing comorbidities, which include metabolic diseases such as hypertension, obesity, diabetes, and vascular diseases. Those with such diseases, or with elevated risk factors for such diseases, will have a compromised endothelium, favoring endothelial dysfunction. The infection of endothelium by SARS-CoV-2 seems to add to this problem, by further damaging the endothelium, causing dysfunction, disruption of vascular integrity, and endothelial cell death. These events lead to the exposure of the thrombogenic basement membrane and result in the activation of the thrombotic and clotting cascade. Because of these observations, critical care clinicians recommend aggressive anti-thrombotic and thrombolytic therapies in the management of acute COVID-19 cases.

\section{Twindemic of COVID-19 and Cardio- metabolic Diseases}

The hospitalization rate for COVID-19 is $4.6 \%$ per 100,000 population, and almost $90 \%$ of the hospitalized patients have some type of underlying condition, according to the Centers for Disease Control and Prevention, USA. According to the Associated Hospitalization Surveillance Network, which includes laboratory-confirmed cases in 99 counties in 14 states, the hospitalization rate increased with patient age. Those aged 65 years and older were admitted at a rate of $13.8 \%$, those aged 50 to 64 at $7.4 \%$, and 18 to 49 at $2.5 \%$. Hypertension was the most common morbidity among the oldest patients, with a prevalence of $72 \%$, followed by cardiovascular disease (CVD) at $50.8 \%$ and obesity at $41 \%$. Is this the common pattern of comorbidity in all geographical areas? Not necessarily. The first large study conducted in the city of New York concluded that pre-existing conditions such as hypertension and diabetes were highly prevalent, and the pattern was similar to the data reported from China. ${ }^{(30)}$ In January of 2020, Huang and associates reported clinical features of 41 patients hospitalized with Covid-19 in Wuhan. ${ }^{(31)}$ The median age was 49 and all were males. According to the authors, less than half had underlying diseases, including diabetes (20\%), hypertension (15\%), and cardiovascular disease $(15 \%)$. Chen and associates from Wuhan's Jinyintan Hospital reported a study of 99 COVID-19 patients. ${ }^{(32,33)}$ The average age was 55 years. Half of the patients had chronic diseases, including cardiovascular and cerebrovascular diseases. Wang and associates from Zhongnan Hospital, Wuhan, reported clinical characteristics of 138 hospitalized patients with the 2019 coronavirus. ${ }^{(34)}$ Of the 138 COVID-19 patients, 64 had one or more coexisting medical conditions; hypertension $(31.2 \%)$, diabetes (10.1\%), and CVD (14.5\%).

A meta-analysis of five studies by cardiologists of Shandong University, China, reported the presence of comorbidities in COVID-19 patients admitted to hospitals. (34) The leading comorbidities were hypertension $(17.1 \%)$, cardiac-cerebrovascular disease (16.4\%), and diabetes $(9.7 \%)$, in that order. ${ }^{(34)}$ Severity and fatality seem to increase with comorbidities such as hypertension, obesity, cardiovascular disease, diabetes, and chronic pulmonary disease. ${ }^{(26-28)}$ In a large study of 72,314 patients from China, the authors reported that those who needed hospitalization had underlying conditions, especially hypertension, diabetes, and cardiovascular disease. ${ }^{(35,36)}$ Early risk assessment - monitoring risk factors for hypoxia, neutrophil extracellular traps, blood vessel damage, lung injury, cardiac injury (cTn1 and proBNP), cytokine storm (IL-6, IL-7, IL-22, IL-17, etc.), and activation of the coagulation cascade cascade (fibrinogen, D-dimer, plasmin) — will help the clinicians in making a wise decision for appropriate interventions.

\section{COVID-19: Hematological Disorders}

The most common hematological findings reported include lymphocytopenia, neutrophilia, eosinopenia, mild thrombocytopenia, less frequently thrombocytosis. ${ }^{(37)}$ According to a meta-analysis, leukocytosis, lymphopenia, and thrombocytopenia are associated with the severity and even fatality of COVID-19 cases. $^{(38)}$ During this disease, changes in hemostasis have also been reported, such as prolonged prothrombin time and activated partial thromboplastin time and increased D-dimer levels. In severe cases of this disease, D-dimer levels seem to get elevated, with the formation of microthrombi in peripheral blood vessels. Furthermore, IL-6, IL-10, and serum ferritin were also strong discriminators for the severity of the disease. Several mediators modulate the release of chemoattractant and neutrophil activity. It is believed that higher values of proinflammatory markers are related to extensive lung injury. ${ }^{(39)}$ The neutrophils are known to develop a sophisticated network of extracellular fibers composed of DNA containing histones, called neutrophil extracellular traps (NETs). There is some evidence to suggest that NETosis is conditional on the production of reactive oxygen species (ROS). Whereas several stimuli trigger NETosis, including pathogen-associated molecular patterns, damage-associated molecular patterns, and inflammatory mediators.

In an earlier article on this topic, we described that neutrophilia predicts poor outcome in patients with severe COVID-19 cases, and neutrophil to lymphocyte ratio may be an independent risk factor for the severity of this disease. ${ }^{(27)}$ In a recent article, Shivakumar with associates from India found that the neutrophil-to-lymphocyte-to monocyte ratio and the platelet-to-lymphocyte ratio were prognostic significant in COVID-19. ${ }^{(40)}$ According to these authors, in inflammation, platelet production increases due to the increased synthesis of thrombopoietin, which is modulated by cytokines. Acute lung injury also leads to leaky blood vessels. Activated platelets enhance lymphocyte adhesion to the endothelium. These events enhance the inflammation, platelet activation, expression of tissue factor, and promote a prothrombotic condition. The elevation of D-dimer, observed in some of the studies, indicates the occurrence of a prothrombotic event, followed by thrombolysis. In a normal situation, the thrombolytic system should clear thrombus formed by endogenous thrombolytic agents. Researchers from the University of North Carolina by infecting Serpine1-knockout mice with SARS-CoV have demonstrated the increased expression of tissue factor and Serpine2 in the absence of Serpine1 and an overall dysfunction of the urokinase pathway. The results of these studies 
demonstrate that a fine balance exists between host coagulation and fibrinolysis pathways regulating pathological disease outcomes, including infection by highly pathogenic viruses such as SARS-CoV-2.(41-43)

\section{COVID-19: Hypertension}

Hypertension is the leading cause of cardiovascular disease and premature death worldwide. ${ }^{(44)}$ Estimates suggest that $31 \%$ of adults ( 1.39 billion) worldwide had hypertension in 2010. These figures just estimate and do not represent reality. Having said that, we assure readers that hypertension is one of the leading metabolic diseases, which significantly contributes to vascular dysfunction, arterial stiffness, hardening of the arteries, and progress of subclinical atherosclerosis.

In the editorial of the American Journal of Hypertension (AJH. 2020;33(5):373), Schiffrin and associates discuss hypertension as one of the most frequently reported comorbidities in patients with COVID-19. They state in their review that the frequency with which COVID-19 patients are hypertensive is not entirely surprising nor does it necessarily imply a causal relationship between hypertension and COVID-19 or its severity, since hypertension is exceedingly frequent in the elderly, and older people appear to be at particular risk of being infected with SARS-CoV-2 virus and of experiencing severe forms of and complications of this disease.

This can be said for all the known metabolic diseases. Early reports suggesting that SARS-CoV-2 binds to ACE2 in the lungs to enter the cells has raised questions about the possibilities that ACE-inhibitors or angiotensin receptor blockers (ARBs) used in the treatment of hypertension may somehow alter the severity of the otherwise of coronavirus disease.

The authors of this editorial concluded, that there is no evidence that hypertension is related to the outcomes of COVID-19, or that ACE-inhibitors or ARBs use is harmful. This editorial in AJH discussed the coronavirus disease more from the treatment of hypertension point of view and did not discuss the role of hypertension as a pre-existing condition that enhanced the severity of the coronavirus disease.

A great number of studies have shown that aging and hypertension are associated with impairments of endotheliumdependent vascular relaxation in the coronary, forearm, and renal arteries, and endothelial dysfunction. ${ }^{(45,46)}$ The normal hemodynamics is maintained by a fine balance between the vasoactive compounds generated between the vessel wall components and the circulating blood components. As an example, in Figure 2, we have shown a schematic representation of the role of vasoactive metabolites of arachidonic acid and L-arginine, generated by the endothelial cells as the mediators of vasodilation.

We also have shown that the synthesis of these endogenous vasodilators is inhibited by lipid hydroperoxides and oxidized lipoproteins formed in the circulating blood. According to Prof. Mehra of Harvard University, the virus enters the lung, destroys the lung tissue, breaks open some blood vessels, then starts to infect endothelial cells, initiates a local immune response, and inflames the endothelium. He further elaborates, "A respiratory virus infecting blood cell, and circulating through the body, is virtually never heard of." The pathophysiology of hypertension involves the complex interaction of multiple vascular effectors, including activation of the sympathetic nervous system, of the renin-angiotensin-aldosterone system, and the inflammatory mediators. Oxidative stress and endothelial dysfunction are consistently observed in hypertensive subjects. COVID-19 patients with hypertension seem to have severe pneumonia, excessive inflammation, compromised endothelium, organ and tissue damage, and severity of the disease. Patients with hypertension should be given additional attention to prevent the added disease burden posed by the pre-existing comorbidity.

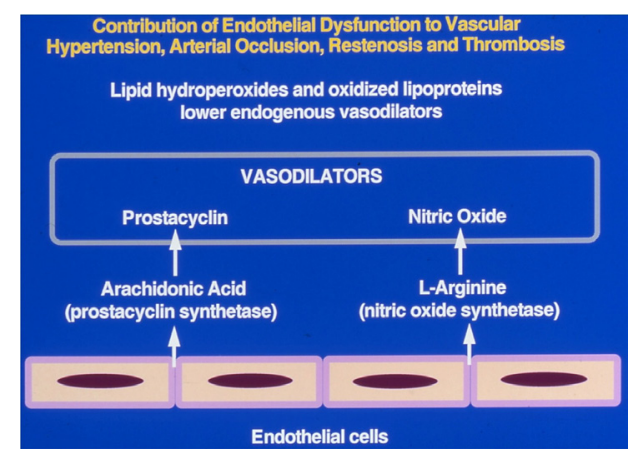

Fig. 2. Contribution of endothelial dysfunction to altered hemodynamics

\section{COVID-19: Excess Weight and Obesity}

According to the World Health Organization, in 2016 more than 1.9 billion adults, 18 years or older, were overweight. Of these over 650 million were obese. Over 340 million children and adolescents, aged 5-19, were overweight or obese. These obese individuals are at a higher risk for coronavirus disease. In the first meta-analysis of its kind, published on 26 August 2020, in Obesity Reviews, researchers pooled data from scores of articles capturing 399,000 patients. They found that people with obesity who contacted SARS-CoV-2 were $113 \%$ more likely than people of healthy weight to land in the hospital, $74 \%$ more likely to be admitted to an ICU, and $48 \%$ more likely to die. ${ }^{(47)}$ People with obesity are prone to have the metabolic disease, with unhealthy levels of blood sugar, fat, and elevated blood pressure. They also seem to have a lower immune response than healthy individuals. Patients with metabolic syndrome, as well as those who are obese have been shown to have reduced response to the vasodilator acetylcholine after intra-arterial infusion, suggesting vascular dysfunction. ${ }^{(48-50)}$

Researchers who reviewed Data from 6916 patient records from Kaiser Permanente reported that compared to normal body mass index (BMI) of $18-24 \mathrm{~kg} / \mathrm{m}^{2}$, the risk of death more than doubled for patients with a BMI of $40-44 \mathrm{~kg} / \mathrm{m}^{2}$ and nearly doubled again, for those with a BMI of $45 \mathrm{~kg} / \mathrm{m}^{2}$ or more. ${ }^{(51)} \mathrm{In}$ an accompanying editorial, David A Kass, a Cardiologist at the Johns Hopkins University, wrote, "that these findings are taken with prior research, should put to rest the contention that obesity is common in severe COVID-19, because it is common in the population." The pathophysiology of hypertension involves the complex interaction of multiple vascular effectors, including activation of the sympathetic nervous system, of the renin- 
angiotensin-aldosterone system, and the inflammatory mediators. ${ }^{(52)}$ Oxidative stress and endothelial dysfunction are consistently observed in hypertensive subjects. ${ }^{(53)}$ As the body weight increases, the blood pressure also rises. Therefore, people with excess weight or obesity will have the added burden of hypertension and clinical manifestation associated with this altered blood vessel function. Each metabolic disease is syndemic in its nature, as it adds additional disease burden to the pre-existing conditions.

As we have discussed earlier, obesity has reached epidemic proportions worldwide. In the USA alone, the prevalence of obesity has increased $50 \%$ in the past three decades, with $70 \%$ of all adults being classified as either overweight or obese. Beyond an impaired response to infections, people with obesity also suffer from chronic, low-grade inflammation. Fat cells secrete inflammation triggering chemical messengers called cytokines, and more come from immune cells called macrophages, that clean up dead and dying fat cells. These, in turn, impair vascular homeostasis and lead to endothelial dysfunction. ${ }^{(54)}$ Data on how to treat COVID-19 patients with obesity are scant. Dr. Scott Khan, an obesity medicine physician at the National Center for Weight and Wellness, suggests giving such patients higher doses of anticoagulants. Looks like it is left to the attending clinicians to develop and administer the best treatment protocol, based on the clinical symptoms presented at the time of admission.

\section{COVID-19: Type-2 Diabetes}

According to a recent article in the journal Science, dozens of studies have reported that many of the sickest COVID-19 patients have been people with obesity. ${ }^{(47)}$ The impact extends to the $32 \%$ of people in the United States who are overweight. The largest descriptive study of hospitalized COVID-19 patients by Genentech researchers found that $77 \%$ of the nearly 17,000 patients hospitalized with CIVID-19 were overweight (29\%) or obese $(48 \%)$. These obese individuals also will have other compounding problems, diabetes being one. It is well known that the blood of people with diabetes has prothrombotic status, meaning, 'increased tendency to clot.' Coronavirus injures endothelial cells, which are already compromised in obese and diabetic individuals, and as such response to this insult by activation of platelet and coagulation pathways. Furthermore, immunity also weakens in people with excess weight, obesity, and diabetes. According to the authors of an article in NEJM, there seems to be a bidirectional relationship between Covid-19 and diabetes. ${ }^{(55)}$ The virus binds to the ACE2 receptors, which are expressed in key metabolic organs and tissues, including pancreatic beta cells, adipose tissue, the small intestine, kidneys. The authors speculate that SARS-CoV-2 may cause pleiotropic alterations of glucose metabolism that could complicate the pathophysiology of preexisting diabetes or its clinical manifestations.

Metabolic diseases such as hypertension, excess weight, and obesity induce vascular remodeling through various mechanisms. Under normal conditions, vascular structure, vessel wall physiology, and flow dynamics are maintained by a variety of endogenous modulators. However, metabolic stress forces the vessel to adapt and remodel depending upon the nature of the stress. Metabolic factors such as hyperglycemia, oxidative stress, and chronic inflammation play a role in diabetes. Since hypertension, excess weight, and obesity contribute significantly to the development of diabetes, major drivers of vascular remodeling are endothelial dysfunction due to altered synthesis of vasodilators and vasoconstrictors, and changes in the complex signaling pathways. ${ }^{(56)}$ Irrespective of whether it is hypertension, excess weight, obesity, or diabetes, the molecular mechanisms involved in the development of altered blood flow, arterial stiffness, hardening of the arteries, and vascular dysfunction seem to be the same. However, in the case of diabetes hyperglycemic state of the blood adds one more contributor to the vascular damage and remodeling. No matter how we look at this situation, all the metabolic diseases have one thing in common, a dysfunctional vascular system, and a compromised immune system, that contributes to the severity of coronavirus disease in these patients.

To address such issues, an international group of leading diabetes researchers participating in the CoviDIAB Project have established a global registry of patients with Covid-19related diabetes. The goal of the registry is to establish the extent and phenotype of new-onset diabetes that is defined by hyperglycemia, confirmed Covid-19, negative history of diabetes, and a history of normal glycated hemoglobin (covidiab.edendrite.com). Since our interest is metabolic diseases, any infectious disease that is syndemic on metabolic risks becomes an important topic of interest. In the countries with the largest number of diabetic individuals, China, India, and the USA, there are an equal number of prediabetic individuals. If we put together pre-diabetics and diabetics, the total number of people at risk for COVID-19 related risk exceeds a billion individuals. Therefore, in our opinion, individuals with hypertension, excess weight, obesity, and diabetes are at a greater risk of severe COVID-19. The question of why China and India with the largest populations of diabetics have relatively low rates of COVID-related mortality is quite puzzling. In China, the COVID-19 pandemic's epicenter, Wuhan, and its province, Hubei, Chinese Center for Disease Control-network, formed 1300 epidemic investigation teams, in addition to the 40,000 doctors and nurses. They used very cleverly tracing tools with big data support. In the first week of January the novel coronavirus infection was detected, and on 23 January 2020, they locked down the city of 11 million people and soon the rest of the Hubei-a province of nearly 60 million. The WHO-China Joint Mission on Coronavirus Disease 2019 Task Force concluded, "In the face of an unknown virus, China has rolled out perhaps the most ambitious, agile, and aggressive, disease containment effort in history." The strategy that underpinned this containment effort was initially a national approach, that promoted universal temperature monitoring, masking, and handwashing. ${ }^{(57)}$ As far as India is concerned, the general population thinks that they have innate immunity, as they are exposed to a variety of Asian viruses, including several strains of coronaviruses. The second surge of COVID-19 in India has demonstrated that those individuals with comorbidities such as hypertension and type 2 diabetes are at higher risk for severe coronavirus disease and death. Yet, COVID-related deaths and case fatality rates reported are relatively low in India.

\section{COVID-19: Cardiac Health}

Novel coronavirus disease seems to affect all the vital organs of the body including the heart. Several studies have 
reported that many COVID-19 survivors experience some type of heart damage, even if they do not have underlying heart disease. "Very early into the pandemic, it was clear that many patients who were hospitalized were showing evidence of cardiac injury," says Dr. Gregg Fonarow, chief of the division of cardiology at the University of California, Los Angeles. He further elaborates on this condition, "This raises concerns that there may be individuals who get through the initial infection but are left with cardiovascular damage and complications." In JAMA Cardiology, analysis of autopsies on 39 COVID-19 patients identified infections in the hearts of patients who had not been diagnosed with cardiovascular issues while they were ill. ${ }^{(58)}$ The novel virus can damage the heart in several ways. For instance, the virus may directly invade the heart muscle; it may indirectly harm the heart by disrupting the balance between oxygen supply and demand. The heart injury may be measured by elevated levels of the enzyme, cardiac troponin in the blood. An elevated level of this cardiac enzyme has been reported in about one-quarter of patients hospitalized with COVID-19. Invasion of the heart muscle may induce myocarditis, which results in an enlarged or weakened heart, low blood pressure, and fluid accumulation in the lungs.

Since the virus uses the ACE2 enzyme as the preferred receptor for entry, the virus may directly invade these receptors on myocardial tissues and cause direct viral harm. It also can affect the heart muscle itself through inflammation, leading to significant heart failure. Dr. Hyung Chun a Yale Cardiologist and director of Translational Research, says one emerging belief is that the endothelial cells, which line the blood vessels, in people with cardiac issues respond differently to the body's immune response. They seem to release inflammatory cytokines that further compromise the body's inflammatory process and lead to the formation of blood clots. The 'inflamed' endothelium seems to contribute to worsening outcomes in COVID-19 and is an important factor contributing to the risk of heart attacks and stroke. As a rule, those confirmed with COVID-19 infection should be on the lookout for symptoms that may indicate damage to the heart or cardiac complications. These symptoms include shortness of breath, chest pain, or heart palpitations. These symptoms may indicate myocarditis, which is one of the potential factors for developing acute coronavirus disease 2019 cardiovascular syndrome (ACovCS). The American Heart Association Journal, Circulation, has published a 'White paper' on this topic. ${ }^{(59)}$ The article reviews the best available data on acute COVID-19 Cardiovascular syndrome epidemiology, pathogenesis, diagnostic, and treatment.

\section{COVID-19: Cardiovascular Disease}

The pre-existing metabolic risks seem to cause worse outcomes and increased risk of death in patients with COVID-19, whereas this virus can also cause vascular damage, myocardial injury, arrhythmia, acute coronary syndrome, and venous thromboembolism. Dr. Nishiga and associates of the Stanford Cardiovascular Institute (CVI), Stanford University School of Medicine, summarized the current understanding of COVID-19 and the cardiovascular system in the journal Nature. ${ }^{(60)}$ As we have discussed earlier, the interaction between the viral spike $(\mathrm{S})$ protein and angiotensin-converting enzyme 2, which facilitates the entry of the virus into host cells, seems to be involved in the cardiovascular manifestations of COVID-19. In one of the earliest reports from Wuhan, involving 41 hospitalized covid-19 patients, the prevalence of comorbidity was greater than $30 \%$ and the most common underlying metabolic diseases were diabetes $(20 \%)$, hypertension (15\%), and CVD (15\%). ${ }^{(2)}$ In a report from Italy involving 1,591 patients with COVID-19 who needed ICU care, 49\% had hypertension, 22\% had CVD, and $17 \%$ had diabetes. ${ }^{(61)}$ Whereas in a report from New York, USA, of the 393 COVID patients who were on ventilators, up to $50 \%$ had hypertension, $36 \%$ had obesity, $25 \%$ had diabetes and $14 \%$ had coronary artery disease. ${ }^{(62)}$ Recent observations suggest that approximately $25 \%$ of people hospitalized with COVID-19 have cardiovascular complications, contributing to about $40 \%$ of all COVID-19-related deaths.

Even though acute hypoxic respiratory failure is the characteristic clinical feature of SARS-CoV-2, many diverse clinical cardiovascular (CV) manifestations have been reported, including heart failure, circulatory shock, cardiomyopathy, arrhythmia, and vascular thrombosis. Since it has been well established that CV-related manifestations portend greater mortality, they pose a great challenge to the clinicians working to develop an appropriate treatment protocol. It becomes essential for the clinicians to think through and consider all possible $\mathrm{CV}$ clinical manifestations, appropriate biomarkers for early diagnosis of these risks, and to develop appropriate therapies for COVID-19-positive patients. ${ }^{(63)}$ According to a white paper published by the American Heart Association editors, the two take-home points are 1) Elevated levels of troponin are frequently seen in patients with COVID-19 disease and are associated with increased severity of disease and risk of death; and 2) In the absence of a specific cause, elevated levels of cardiac troponins are likely attributable to myocardial injury from inflammation or a direct effect of SARS-CoV-2 infection. ${ }^{(64)}$ The paper also poses some questions that need to be addressed. 1) The role of troponin in clinical risk stratification, and as a prognostic factor of disease severity and mortality. 2) Mechanistic studies are needed to evaluate the cause of myocardial injury and determine whether there is a potential for a therapeutic option. Many studies have reported that infection with COVID-19 may predispose one to venous and arterial thromboembolism to a greater degree with worse disease severity. ${ }^{(65)}$ The management of CVD in patients with COVID-19 includes general supportive treatment, circulatory support, other symptomatic treatment, and psychological assistance, as well as online consultation.

\section{COVID-19: Cerebrovascular Disease}

In one of the early studies reported from Wuhan, China, researchers conducted a retrospective study of 214 patients admitted with COVID-19 infection. Of these (mean age of 52) 78 patients $(36.4 \%)$ had neurologic manifestations. ${ }^{(66)}$ SARS-CoV-2 binds to ACE-2, leading to the downregulation of this receptor function and contributes to the post-ischemic inflammation cascade resulting in decreased perfusion of the ischemic areas of cerebral tissue with larger infarct volume and ischemic stroke. ${ }^{(67)}$ Furthermore, ACE2 dysfunction may 
cause hypertensive peaks and impairment of cerebrovascular endothelium, leading to intracerebral hemorrhage. It has also been reported that systemic hypotension may induce hypo ischemic encephalopathy and microbleeds. Researchers from Saudi Arabia report that neurological complications range from dizziness, headache, hyposmia, encephalopathy, infarcts, microhemorrhages, and stroke. ${ }^{(68)}$ They also report decreased deformability of red blood cells and hypercoagulability, resulting in hypoxia and inflammation in these patients. A systematic review has analyzed data from 1,210 articles with 226 cases of ischemic stroke. ${ }^{(69,70)}$ According to this report, large vessel occlusion (LVO) was observed in 105 patients, acute intracranial bleeding in 35 patients, intracerebral hemorrhage (ICH) in 24 patients, 4 patients had non-traumatic subarachnoid hemorrhage (SAH), remaining 7 patients had simultaneous presence of SAH and ICH. The authors concluded that cerebrovascular events are relatively common findings in COVID-19 infection and could have a multifactorial etiology.

Furthermore, of the total of 226 cases of ischemic stroke, 35 cases of intracranial bleeding, and 14 cases of cerebrovascular sinus thrombosis (CVST), were reported. According to these investigators, postmortem brain magnetic resonance imaging (MRI) showed extensive signs of cerebrovascular involvement, including microbleeds with subcortical and posterior predominance. Among these patients, D-dimer levels were at least four-fold higher than normal values. Inflammation is a common occurrence in Covid-19 patients. It is well known that both acute and chronic infections and inflammatory states serve as triggers of stroke. By and large, COVID-19 patients with preexisting metabolic diseases such as hypertension, excess weight, obesity, type-2 diabetes, and CVDs have increased severity at the time of admission to the hospital. These pre-existing risks make it very difficult to develop an optimal treatment regimen.

\section{COVID-19: Arterial and Venous Diseases}

Coronavirus disease severity is associated with venous thromboembolism as well as arterial thrombosis. Thrombotic complications, as well as coagulopathy, have been reported in COVID-19 positive individuals. COVID-19 associated coagulopathy (CAC), detected by the increase in D-dimer and fibrinogen levels with minimal abnormalities in prothrombin time and platelet count has been reported in COVID-positive cases. The high mortality associated with thromboembolic diseases in COVID-19 has prompted clinicians to use D-dimer as a useful marker for assessing the severity of the disease. ${ }^{(71)}$ According to a review by Harvard Medical School researchers, in critically ill patients with COVID-19, elevated levels of D-dimer were found in 100\% of participants, elevated fibrinogen in $74 \%$ of the participants, and factor V11 in 100\% of participants. Antiphospholipid antibodies were detected in $53 \%$ of the participants, decreased protein $\mathrm{C}$, protein $\mathrm{S}$, and antithrombin levels were detected in all participants. ${ }^{(72)}$ Coagulation abnormalities were associated with acute vascular events such as stroke, peripheral arterial ischemia, and venous thromboembolism. Guidelines from the American College of Chest Physicians suggest prophylaxis with Low Molecular Weight Heparins (40mg) and fondaparinux $2.5 \mathrm{mg}$, in the absence of any contraindications, such as active bleeding. However, this professional society does not recommend post-discharge thromboprophylaxis. The International Society on Thrombosis and Hemostasis suggests duration of 14 to 30 days for postdischarge thromboprophylaxis. Thromboprophylaxis for patients who do not require hospitalization is not currently recommended.

\section{Prevention}

When discussing prevention strategies, we need to consider the unprecedented pandemic of SARS-CoV-2 as well as a pre-existing epidemic of metabolic risks, metabolic diseases, and vascular diseases worldwide, which increase the severity of COVID-19. Having said that, we need to remember that no country has reduced, reversed, or prevented the increase in the incidence of metabolic diseases. Metabolic diseases have increased rapidly to epidemic proportions worldwide in the last three decades. COVID-19 is not going to go away any sooner. Twindemic is going to continue to play havoc for quite some time to come and will be a public health experts' nightmare. In a large study of 72,314 patients from China, the authors reported that those who needed hospitalization had underlying conditions, especially hypertension, obesity, diabetes, and cardiovascular disease. ${ }^{(35,36)}$ Early risk assessment, monitoring risk factors for hypoxia, neutrophil extracellular traps (NETs), blood vessel damage, lung injury, platelet activation, the formation of microthrombi or microbleeds, cardiac injury (cTn1 and proBNP), cytokine storm (IL-6, IL-7, IL-22, IL-17, etc.), activation of the coagulation cascade (fibrinogen, D-dimer, plasmin), will help the clinicians in making a wise decision for appropriate interventions. ${ }^{(73,74)}$

In the absence of a cure for coronavirus disease, sensible medicine proposes a gentler, moderate, and humble view of available treatment options and their effectiveness in patients with COVID-19. The approach encourages clinicians, to elevate usual care, reduce unnecessary interventionism, focus, and rely on scientific rigor. By and large, treatment options are based on clinical diagnosis-based treatments for observed symptoms. For patients with COVID-19, who are not hospitalized or who are hospitalized with moderate disease, but do not require supplemental oxygen, the National Institutes of Health (NIH, USA) panel does not recommend any specific antiviral or immunomodulatory therapy, for the treatment of coronavirus disease in these patients. For those hospitalized with severe conditions, the panel recommends Remedesivir $200 \mathrm{mg}$ intravenously (IV) for 1 day followed by a $100 \mathrm{mg}$ dose for four days or until hospital discharge. A combination of Remedesivir and dexamethasone $6 \mathrm{mg}$ IV up to 10 days. As mentioned earlier, currently, there are no US FDA-approved therapies, for coronavirus disease treatment. FDA has created a special emergency program for possible coronavirus therapies, the Coronavirus Treatment Acceleration Program (CTAP). Currently, there are 590 drug development programs in planning stages, 390 trials in review, and five authorized for emergency use, none approved for use in COVID-19 management.

As of this writing, there are at least 51 studies listed in the COVID-19 vaccine tracker of the Regulatory Affairs Professional Society (RAPS) site. The top ten entries, which are 
under phase 3 trial, include $\mathrm{Ad} 5-\mathrm{nCoV}$, a recombinant vaccine by CanSino Biologics (China); AZD1222, a replicationdeficient adenovirus vector vaccine (The University of Oxford, the Jenner Institute); CoronaVac by Sinovac; JNJ-78436735, a non-replicating viral vector by Johnson and Johnson; mRNA1273, an mRNA-based vaccine by Moderna; an unnamed inactivated vaccine by Wuhan Institute of Biological Products; NVX-CoV2373, a nanoparticle vaccine by Novavax. There are several new entries in phase $2 / 3$ trials, including the BCG vaccine by the University of Melbourne and Mass. General Hospital, Boston; BNT162, an mRNA-based vaccine by PfizerBioNTech; and Covaxin, an inactivated virus vaccine by Bharat Biotech, National Institute of Virology, India. According to a recent article in the New Engl. J. Med., confidence in any COVID-19 vaccine that is made available under an emergency use authorization (EUA) will depend on the rigor of the clinical criteria, including the duration of follow-up, safety, and efficacy of the vaccine. ${ }^{(75)}$ With phase 3 clinical trials of COVID-19 vaccine underway, safety and efficacy data will be provided to the FDA soon after they are compiled. ${ }^{(76)}$ Emergency use authorization will be made by the FDA's Center for Biologics Evaluation and Research (CBER). The decision of this branch of the FDA has been approved by the Vaccines and Related Biological Products Advisory Committee (VRBPAC).

There are twelve vaccines against SARS-CoV-2 that have been authorized for use in various locations around the world. These include mRNA vaccines, viral vector vaccines, subunit vaccines, and inactivated virus vaccines. mRNA vaccines do not contain any part of the virus. They carry a chemically synthesized piece of messenger RNA, containing the information needed for cells to make the spike proteins of the virus that is essential for infection. Like the mRNA, vector vaccines also do not contain the SARS-Cov-2 virus. They use adenovirus as the delivery system. Subunit vaccines use part (spike protein) of the SARS-Cov-2 virus. Inactivated vaccines contain the entire SARS-CoV-2 virus inactivated by betapropiolactone. At the time of this writing, globally, the following vaccines have been given emergency use authorization against COVID-19: mRNA-1273 (Moderna, US), BNT162b2 (PfizerBioNTech, US), Ad26CoV2.S (Viral vector, Johnson \& Johnson, US), ChAdOx1(Viral vector, AstraZeneca/Oxford, UK), NVX-CoV2373 (Novavax Inc, US), CVnCoV-mRNA (GSK, Germany), Gam-COVID-Vac (Sputnik V, Viral vector, Russia), CoronaVac (inactivated virus, China), and BBIBPCorV (inactivated virus, China), Covaxin (inactivated virus, Bharath Biotech, India). ${ }^{(77)}$

A variety of nanobodies (nAbs) against SARS-CoV-2, which target the $\mathrm{S}$ protein of the virus, are in preclinical development. ${ }^{(78)}$ Monoclonal antibodies are usually large and must be produced in mammalian cell expression systems. On the other hand, nanobodies are single-domain antibodies with small size, good solubility, stability and could be produced in Escherichia coli and yeast cell cultures. Therefore, they could be produced at relatively low cost in large quantities. National Institutes of Health researchers have isolated tiny antibodies against SARS$\mathrm{CoV}-2$. One of the advantages of the nanoantibodies is that they can be aerosolized to use in inhalers to coat the lungs and airways, the preferred route of SARS-CoV-2 entry.
Considering the contribution of comorbidities to the progression and severity of the coronavirus disease, one would expect that China, India, and the USA, with the largest populations of diabetic subjects, should have the highest CFR (Deaths per 100,000 population) for COVID-19. On the other hand, Mexico (8.5\%), Peru (3.6\%), Italy (3.5\%), and South Africa have a lot more mortality than the USA (1.0\%) and India (1.7\%) (Coronavirus.jhu.edu/data/mortality; February 4, 2021). Since the three major populations with the highest number of diabetics have not shown a comparatively high case fatality rate, it is worthwhile discussing the other two comorbidities (hypertension and obesity) as the chief contributors for the Covid-19 progression and severity. Trends in the prevalence of hypertension in the USA, according to the NHANES survey of age-standardized prevalence, decreased from $48.4 \%$ in 1999 2000 to $45.4 \%$ in $2015-2016$. However, the absolute burden of hypertension consistently increased, from 87.0 million in 19992000 to 108 million in 2015-2016. ${ }^{(79)}$ Hypertension appears to be more common in Mexico, than among Mexican Immigrants in the United States. As far as obesity goes, the number of obese children and adolescents aged five to 19 years, has risen tenfold in the past four decades and if current trends continue, there will be more obese children and adolescents than those moderately. ${ }^{(80)}$ Among adolescents, obesity prevalence in the USA was $16.8 \%$ in 2007 and $18.5 \%$ in 2016. Age-standardized obesity in adults increased from $33.7 \%$ in 2007 to $39.6 \%$ in 2015. Whereas $62 \%$ of the participants in Mexico reported, at least, being overweight. ${ }^{(81)}$ When considering obesity data based on the BMI, we should keep in mind that South Asians have a different body fat distribution, compared to the European and Western populations. South Asians in general have central abdominal obesity.

If we carefully analyze a series of clinical events, that develop a post-SARS-CoV-2 infection, we can begin to understand, why metabolic diseases serve as independent risk factors for the progression and severity of coronavirus disease. The initial route of entry is via the nasal and oral mucosa, the preferred receptor that facilitates the transmission seems to be the ubiquitous ACE2, which is found in multiple types of cells and tissue including vascular endothelium. Recent findings, that following the injury to the lung tissue, the virus gets entry into the endothelium, opens a whole new avenue for the progress of the disease and its severity. The endothelium is the largest organ of the body, covering a large surface area and reaching out to every tissue and organ. As such, the injury to the endothelium could introduce a cascade of events, leading to platelet activation, thrombin generation, and promotion of both thrombotic and thrombolytic events. ${ }^{(29,41)}$ Furthermore, people with metabolic diseases such as hypertension, excess weight, obesity, diabetes (Type 2), and vascular disease, already have a compromised endothelium, and invasion of the SARS-CoV-2 virus leads to endothelialitis and causes further injury to the vascular system, disruption of vascular integrity and endothelial cell death. ${ }^{(82-85)}$ These events lead to the exposure of the subendothelial basement membrane and result in the activation of thrombotic and clotting cascade of events.

This unprecedented pandemic of SARS-CoV-2 has taught us two lessons: first, the importance of robust public 
health infrastructure, and second, the need for an immediate call for action to reduce, reverse, or prevent metabolic diseases worldwide. The recent statement from the Institute for Fiscal Studies Deaton Review on inequality (IFS Deaton Review, Jan 5, 2021), stressed that the opportunity cost of the pandemic for young people is potentially huge, but also that it is a "once-ina-generation opportunity to tackle the disadvantages faced by many that this pandemic has so devastatingly exposed." In a recent editorial of the Lancet Public Health, the editor concludes that adopting a broadened, equity-based approach to population health should be an essential part of building a more resilient society that is better prepared for future pandemics. ${ }^{(86)}$

\section{References}

1. Zhu N, Zhang D, Wang W, Li X, Yang B, Song J, et al.; China Novel Coronavirus Investigating and Research Team. A Novel Coronavirus from Patients with Pneumonia in China, 2019. N Engl J Med. 2020 Feb 20;382(8):727-733. doi: 10.1056/ NEJMoa2001017.

2. Huang C, Wang Y, Li X, Ren L, Zhao J, Hu Y, et al. Clinical features of patients infected with 2019 novel coronavirus in Wuhan, China. Lancet. 2020 Feb 15;395(10223):497-506. doi: 10.1016/S0140-6736(20)30183-5.

3. Chen N, Zhou M, Dong X, Qu J, Gong F, Han Y, et al. Epidemiological and clinical characteristics of 99 cases of 2019 novel coronavirus pneumonia in Wuhan, China: a descriptive study. Lancet. $2020 \mathrm{Feb}$ 15;395(10223):507-513. doi: 10.1016/ S0140-6736(20)30211-7.

4. Wang D, Hu B, Hu C, Zhu F, Liu X, Zhang J, et al. Clinical Characteristics of 138 Hospitalized Patients With 2019 Novel Coronavirus-Infected Pneumonia in Wuhan, China. JAMA. 2020 Mar 17;323(11):1061-1069. doi: 10.1001/jama.2020.1585. Erratum in: JAMA. 2021 Mar 16;325(11):1113. PMID: 32031570; PMCID: PMC7042881.

5. Wu C, Chen X, Cai Y, Xia J, Zhou X, Xu S, et al. Risk Factors Associated With Acute Respiratory Distress Syndrome and Death in Patients With Coronavirus Disease 2019 Pneumonia in Wuhan, China. JAMA Intern Med. $2020 \mathrm{Jul}$ 1;180(7):934-943. doi: 10.1001/jamainternmed.2020.0994. Erratum in: JAMA Intern Med. 2020 Jul 1;180(7):1031. PMID: 32167524; PMCID: PMC7070509.

6. Kamps BS, Hoffmann C: COVID REFERENC ENG/2021.6.covidreference.com https:/amedeo.com/ CovidReference06.pdf (www.CovdiRefrence.com)

7. Karabag SF: An unprecedented Global Crisis! The Global, Regional, National, Political, Economic and Commercial Impact of the Coronavirus Pandemic. J App. Econ. \& Bus. Res. 10)10):1-6 (2020)

8. Shrestha N, Shad MY, Ulvi O, Khan MH, KaramehicMuratovic A, Nguyen UDT, et al. The impact of COVID-19 on globalization. One Health. 2020 Dec 20;11:100180. doi: 10.1016/j.onehlt.2020.100180.

9. Haghani M, Bliemer MCJ. Covid-19 pandemic and the unprecedented mobilisation of scholarly efforts prompted by a health crisis: Scientometric comparisons across SARS, MERS and 2019-nCoV literature. Scientometrics. 2020 Sep 21:1-32. doi: 10.1007/s11192-020-03706-z.

10. Cutler DM, Summers LH. The COVID-19 Pandemic and the \$16 Trillion Virus. JAMA. 2020 Oct 20;324(15):1495-1496. doi: 10.1001/jama.2020.19759.

11. Conti P, Caraffa A, Gallenga CE, Kritas SK, Frydas I,
Younes A, et al. The British variant of the new coronavirus-19 (Sars-Cov-2) should not create a vaccine problem. J Biol Regul Homeost Agents. 2021 Jan-Feb;35(1):1-4. doi: 10.23812/21-3E. PMID: 33377359.

12. Horton R: COVID-19 is not a pandemic. Lancet 2020; 396 (10255): 874.

13. Singer M, Bulled N, Ostrach B, Mendenhall E. Syndemics and the biosocial conception of health. Lancet. 2017 Mar 4;389(10072):941-950. doi: 10.1016/S0140-6736(17)30003-X. PMID: 28271845.

14. Mendenhall E. Beyond Comorbidity: A Critical Perspective of Syndemic Depression and Diabetes in Cross-cultural Contexts. Med Anthropol Q. 2016 Dec;30(4):462-478. doi: 10.1111/maq. 12215 .

15. Rao GHR: Global Syndemic of Metabolic Diseases: Editorial Comments. Journal of Diabetes \& Clin. Res. 2018; I(1): 2-4.

16. Rao GHR: COVID-19 and Cardiometabolic Diseases.: Guest Editorial. EC Cardiol 2020; 7.6:08-12.

17. Rao GHR: Coronavirus (COVID-19), Comorbidities, and Acute Vascular Events; Guest Editorial. ECCMC EC Clinical Case Reports. 2020; 3.6:87-91

18. Rao GHR: Coronavirus Disease (Covid-19), Comorbidities, and Clinical Manifestations. Guest Editorial. EC Diab, Met Res. 2020; 4.6 : 27-33.

19. Gussow AB, Auslander N, Faure G, Wolf YI, Zhang F, Koonin EV. Genomic determinants of pathogenicity in SARSCoV-2 and other human coronaviruses. bioRxiv [Preprint]. 2020 Apr 9:2020.04.05.026450. doi: 10.1101/2020.04.05.026450. Update in: Proc NatlAcad Sci USA. 2020 Jun 30;117(26):1519315199.

20. Shang J, Wan Y, Luo C, Ye G, Geng Q, Auerbach A, Li F. Cell entry mechanisms of SARS-CoV-2. Proc Natl Acad Sci U S A. 2020 May 26;117(21):11727-11734. doi: 10.1073/ pnas. 2003138117.

21. Tortorici MA, Veesler D. Structural insights into coronavirus entry. Adv Virus Res. 2019;105:93-116. doi: 10.1016/bs.aivir.2019.08.002.

22. Hoffmann M, Kleine-Weber H, Schroeder S, Krüger N, Herrler T, Erichsen S, et al. SARS-CoV-2 Cell Entry Depends on ACE2 and TMPRSS2 and Is Blocked by a Clinically Proven Protease Inhibitor. Cell. 2020 Apr 16;181(2):271-280.e8. doi: 10.1016/j.cell.2020.02.052.

23. Ming Y, Qiang L. Involvement of Spike Protein, Furin, and ACE2 in SARS-CoV-2-Related Cardiovascular Complications. SN Compr Clin Med. 2020 Jul 11:1-6. doi: 10.1007/s42399020-00400-2.

24. Noor FM, Islam M: Prevalence of Clinical Manifestations and Comorbidities of Coronavirus (COVID-19) Infection: A Meta-Analysis. Fortune J. Health Sci 3 (2020):55-97.

25. Rao GHR. Coronavirus Disease and Acute Vascular Events. Clin Appl Thromb Hemost. 2020 JanDec;26:1076029620929091. doi: 10.1177/1076029620929091. 26. Rao GHR: COVID-19 and Cardiometabolic Diseases.: Guest Editorial. EC Cardiol 2020; 7.6:08-12.

27. Rao GHR: Coronavirus (COVID-19), Comorbidities, and Acute Vascular Events; Guest Editorial. ECCMC EC Clinical Case Reports. 2020;3.6:87-91.

28. Coronavirus Disease (Covid-19), Comorbidities, and Clinical Manifestations. Guest Editorial. EC Diab, Met Res. 2020;4.6:27-33.

29. Rao GHR: Coronavirus Disease (Covid-19): A Disease of the Vascular Endothelium. Series Cardiology Res 2020; 2(1): 23-27. 
30. Richardson S, Hirsch JS, Narasimhan M, Crawford JM, McGinn T, Davidson KW; the Northwell COVID-19 Research Consortium, Barnaby DP, Becker LB, Chelico JD, Cohen SL, Cookingham J, Coppa K, et al. Presenting Characteristics, Comorbidities, and Outcomes Among 5700 Patients Hospitalized With COVID-19 in the New York City Area. JAMA. 2020 May 26;323(20):2052-2059. doi: 10.1001/jama.2020.6775. Erratum in: JAMA. 2020 May 26;323(20):2098.

31. Huang C, Wang Y, Li X, Ren L, Zhao J, Hu Y, et al. Clinical features of patients infected with 2019 novel coronavirus in Wuhan, China. Lancet. 2020 Feb 15;395(10223):497-506. doi: 10.1016/S0140-6736(20)30183-5. Epub 2020 Jan 24. Erratum in: Lancet. 2020 Jan 30;: PMID: 31986264; PMCID: PMC7159299.

32. Chen N, Zhou M, Dong X, Qu J, Gong F, Han Y,et al. Epidemiological and clinical characteristics of 99 cases of 2019 novel coronavirus pneumonia in Wuhan, China: a descriptive study. Lancet. 2020 Feb 15;395(10223):507-513. doi: 10.1016/ S0140-6736(20)30211-7.

33. Wu C, Yang W, Wu X, Zhang T, Zhao Y, Ren W, Xia J. Clinical Manifestation and Laboratory Characteristics of SARS-CoV-2 Infection in Pregnant Women. Virol Sin. 2020 Jun;35(3):305-310. doi: 10.1007/s12250-020-00227-0.

34. Wang B, Li R, Lu Z, Huang Y. Does comorbidity increase the risk of patients with COVID-19: evidence from metaanalysis. Aging (Albany NY). 2020 Apr 8;12(7):6049-6057. doi: 10.18632 /aging. 103000 .

35. Wu Z, McGoogan JM. Characteristics of and Important Lessons From the Coronavirus Disease 2019 (COVID-19) Outbreak in China: Summary of a Report of 72314 Cases From the Chinese Center for Disease Control and Prevention. JAMA. 2020 Apr 7;323(13):1239-1242. doi: 10.1001/jama.2020.2648. 36. Adão R, Guzik TJ. Inside the heart of COVID-19. Cardiovasc Res. 2020 May 1;116(6):e59-e61. doi: 10.1093/cvr/ cvaa086.

37. Letícia de Oliveira Toledo S, Sousa Nogueira L, das Graças Carvalho M, Romana Alves Rios D, de Barros Pinheiro M. COVID-19: Review and hematologic impact. Clin Chim Acta. 2020 Nov;510:170-176. doi: 10.1016/j.cca.2020.07.016.

38. Henry BM, de Oliveira MHS, Benoit S, Plebani M, Lippi G. Hematologic, biochemical and immune biomarker abnormalities associated with severe illness and mortality in coronavirus disease 2019 (COVID-19): a meta-analysis. Clin Chem Lab Med. 2020 Jun 25;58(7):1021-1028. doi: 10.1515/ cclm-2020-0369.

39. Borges L, Pithon-Curi TC, Curi R, Hatanaka E. COVID-19 and Neutrophils: The Relationship between Hyperinflammation and Neutrophil Extracellular Traps. Mediators Inflamm. 2020 Dec 2;2020:8829674. doi: 10.1155/2020/8829674.

40. Bg S, Gosavi S, Ananda Rao A, Shastry S, Raj SC, Sharma A, Suresh A, Noubade R. Neutrophil-to-Lymphocyte, Lymphocyte-to-Monocyte, and Platelet-to-Lymphocyte Ratios: Prognostic Significance in COVID-19. Cureus. 2021 Jan 11;13(1):e12622. doi: 10.7759/cureus.12622.

41. Varga Z, FlammerAJ, Steiger P, Haberecker M,AndermattR, Zinkernagel AS, et al. Endothelial cell infection and endotheliitis in COVID-19. Lancet. 2020 May 2;395(10234):1417-1418. doi: 10.1016/S0140-6736(20)30937-5.

42. Ackermann M, Verleden SE, Kuehnel M, Haverich A, Welte T, Laenger F, et al. Pulmonary Vascular Endothelialitis, Thrombosis, and Angiogenesis in Covid-19. N Engl J Med. 2020 Jul 9;383(2):120-128. doi: 10.1056/NEJMoa2015432. 43. Gralinski LE, Bankhead A3rd, Jeng S, Menachery VD, Proll
S, Belisle SE, et al. . Mechanisms of severe acute respiratory syndrome coronavirus-induced acute lung injury. mBio. 2013 Aug 6;4(4):e00271-13. doi: 10.1128/mBio.00271-13.

44. Mills KT, Stefanescu A, He J. The global epidemiology of hypertension. Nat Rev Nephrol. 2020 Apr;16(4):223-237. doi: 10.1038/s41581-019-0244-2.

45. Higashi Y, Kihara Y, Noma K. Endothelial dysfunction and hypertension in aging. Hypertens Res. 2012 Nov;35(11):103947. doi: 10.1038/hr.2012.138.

46. Panza JA, Quyyumi AA, Brush JE Jr, Epstein SE. Abnormal endothelium-dependent vascular relaxation in patients with essential hypertension. N Engl J Med. 1990 Jul 5;323(1):22-7. doi: 10.1056/NEJM199007053230105.

47. Wadman M: Why COVID-19 is more deadly in people with obesity-even if they are young? Science 2020; doi:10.1126/ science.abe 7010 .

48. Ignarro LJ, Buga GM, Wood KS, Byrns RE, Chaudhuri G. Endothelium-derived relaxing factor produced and released from artery and vein is nitric oxide. Proc Natl Acad Sci U S A. 1987 Dec;84(24):9265-9. doi: 10.1073/pnas.84.24.9265.

49. Van Guilder GP, Hoetzer GL, Dengel DR, Stauffer BL, DeSouza CA. Impaired endothelium-dependent vasodilation in normotensive and normoglycemic obese adult humans. $\mathrm{J}$ Cardiovasc Pharmacol. 2006 Feb;47(2):310-3. doi: 10.1097/01. fjc.0000205097.29946.d3. PMID: 16495771.

50. Steinberg HO, Chaker H, Leaming R, Johnson A, Brechtel $\mathrm{G}$, Baron AD. Obesity/insulin resistance is associated with endothelial dysfunction. Implications for the syndrome of insulin resistance. J Clin Invest. 1996 Jun 1;97(11):2601-10. doi: 10.1172/JCI118709.

51. Tartof SY, Qian L, Hong V, Wei R, Nadjafi RF, Fischer $\mathrm{H}$, et al. Obesity and Mortality Among Patients Diagnosed With COVID-19: Results From an Integrated Health Care Organization. Ann Intern Med. 2020 Nov 17;173(10):773-781. doi: 10.7326/M20-3742.

52. Kass DA. COVID-19 and Severe Obesity: A Big Problem? Ann Intern Med. 2020 Nov 17;173(10):840-841. doi: 10.7326/ M20-5677.

53. Schulz E, Gori T, Münzel T. Oxidative stress and endothelial dysfunction in hypertension. Hypertens Res. 2011 Jun;34(6):665-73. doi: 10.1038/hr.2011.39.

54. Iantorno M, Campia U, Di Daniele N, Nistico S, Forleo GB, Cardillo C, Tesauro M. Obesity, inflammation and endothelial dysfunction. J Biol Regul Homeost Agents. 2014 Apr-Jun;28(2):169-76.

55. Rubino F, Amiel SA, Zimmet P, Alberti G, Bornstein S, Eckel RH, et al. New-Onset Diabetes in Covid-19. N Engl J Med. 2020 Aug 20;383(8):789-790. doi: 10.1056/NEJMc2018688.

56. Gerrard JM, Stuart MJ, Rao GH, Steffes MW, Mauer SM, Brown DM, White JG. Alteration in the balance of prostaglandin and thromboxane synthesis in diabetic rats. J Lab Clin Med. 1980 Jun;95(6):950-8. PMID: 6445927.

57. Report of the WHO-China Joint Mission on Coronavirus Disease 2019 (COVID-19). 16-14 February 2020. The joint Mission consisted of 25 national and international experts from China, Germany, Japan, Korea, Russia, Singapore and the United States of America, and the World Health Organization (WHO).

58. Lindner D, Fitzek A, Bräuninger H, Aleshcheva G, Edler $\mathrm{C}$, Meissner K, et al. Association of Cardiac Infection With SARS-CoV-2 in Confirmed COVID-19 Autopsy Cases. JAMA Cardiol. 2020 Nov 1;5(11):1281-1285. doi: 10.1001/ jamacardio.2020.3551. 
59. Hendren NS, Drazner MH, Bozkurt B, Cooper LT Jr. Description and Proposed Management of the Acute COVID-19 CardiovascularSyndrome.Circulation.2020Jun9;141(23):19031914. doi: 10.1161/CIRCULATIONAHA.120.047349.

60. Nishiga M, Wang DW, Han Y, Lewis DB, Wu JC. COVID-19 and cardiovascular disease: from basic mechanisms to clinical perspectives. Nat Rev Cardiol. 2020 Sep;17(9):543558. doi: 10.1038/s41569-020-0413-9.

61. Grasselli G, Zangrillo A, Zanella A, Antonelli M, Cabrini L, Castelli A, et al.; COVID-19 Lombardy ICU Network. Baseline Characteristics and Outcomes of 1591 Patients Infected With SARS-CoV-2 Admitted to ICUs of the Lombardy Region, Italy. JAMA. 2020 Apr 28;323(16):1574-1581. doi: 10.1001/ jama.2020.5394.

62. Goyal P, Choi JJ, Pinheiro LC, Schenck EJ, Chen R, Jabri A, et al. Clinical Characteristics of Covid-19 in New York City. N Engl J Med. 2020 Jun 11;382(24):2372-2374. doi: 10.1056/ NEJMc2010419.

63. Mahenthiran AK, Mahenthiran AK, Mahenthiran J. Cardiovascular system and COVID-19: manifestations and therapeutics. Rev Cardiovasc Med. 2020 Sep 30;21(3):399409. doi: $10.31083 /$ j.rcm.2020.03.124.

64. Gupta AK, Jneid H, Addison D, Ardehali H, Boehme AK, Borgaonkar S, et al. Current Perspectives on Coronavirus Disease 2019 and Cardiovascular Disease: A White Paper by the JAHA Editors. J Am Heart Assoc. 2020 Jun 16;9(12):e017013. doi: 10.1161/JAHA.120.017013.

65. Klok FA, Kruip MJHA, van der Meer NJM, Arbous MS, Gommers DAMPJ, Kant KM, et al. Incidence of thrombotic complications in critically ill ICU patients with COVID-19. Thromb Res. 2020 Jul;191:145-147. doi: 10.1016/j. thromres.2020.04.013.

66. Mao L, Jin H, Wang M, Hu Y, Chen S, He Q, et al. Neurologic Manifestations of Hospitalized Patients With Coronavirus Disease 2019 in Wuhan, China. JAMA Neurol. 2020 Jun 1;77(6):683-690. doi: 10.1001/jamaneurol.2020.1127. 67. Tsivgoulis G, Palaiodimou L, Zand R, Lioutas VA, Krogias C, Katsanos AH, et al. COVID-19 and cerebrovascular diseases: a comprehensive overview. Ther Adv Neurol Disord. 2020 Dec 8;13:1756286420978004. doi: 10.1177/1756286420978004.

68. Akhter N, Ahmad S, Alzahrani FA, Dar SA, Wahid M, Haque S, et al. Impact of COVID-19 on the cerebrovascular system and the prevention of RBC lysis. Eur Rev Med Pharmacol Sci. 2020 Oct;24(19):10267-10278. doi: 10.26355/ eurrev_202010_23251.

69. Fraiman P, Godeiro Junior C, Moro E, Cavallieri F, Zedde M. COVID-19 and Cerebrovascular Diseases: A Systematic Review and Perspectives for Stroke Management. Front Neurol. 2020 Nov 5;11:574694. doi: 10.3389/fneur.2020.574694.

70. Lee MH, Perl DP, Nair G, Li W, Maric D, Murray H, et al. Microvascular Injury in the Brains of Patients with Covid-19. N Engl J Med. 2021 Feb 4;384(5):481-483. doi: 10.1056/ NEJMc2033369.

71. Guan WJ, Ni ZY, Hu Y, Liang WH, Ou CQ, He JX, et al.; China Medical Treatment Expert Group for Covid-19. Clinical Characteristics of Coronavirus Disease 2019 in China. N Engl J Med. 2020 Apr 30;382(18):1708-1720. doi: 10.1056/ NEJMoa2002032.

72. Piazza G, Morrow DA. Diagnosis, Management, and Pathophysiology of Arterial and Venous Thrombosis in COVID-19. JAMA. 2020 Dec 22;324(24):2548-2549. doi: 10.1001/jama.2020.23422.

73. Bikdeli B, Madhavan MV, Jimenez D, Chuich T, Dreyfus I,
Driggin E, et al.; Global COVID-19 Thrombosis Collaborative Group, Endorsed by the ISTH, NATF, ESVM, and the IUA, Supported by the ESC Working Group on Pulmonary Circulation and Right Ventricular Function. COVID-19 and Thrombotic or Thromboembolic Disease: Implications for Prevention, Antithrombotic Therapy, and Follow-Up: JACC State-of-theArt Review. J Am Coll Cardiol. 2020 Jun 16;75(23):2950-2973. doi: 10.1016/j.jacc.2020.04.031.

74. Rao GHR: SARS-CoV-2 biochemistry, Transmission, Clinical Manifestations, and Prevention. Int J. Biomed. 2020;10(4):303-311.

75. Krause PR, Gruber MF. Emergency Use Authorization of Covid Vaccines - Safety and Efficacy Follow-up Considerations. N Engl J Med. 2020 Nov 5;383(19):e107. doi: 10.1056/ NEJMp2031373.

76. Schwartz JL. Evaluating and Deploying Covid-19 Vaccines - The Importance of Transparency, Scientific Integrity, and Public Trust. N Engl J Med. 2020 Oct 29;383(18):1703-1705. doi: 10.1056/NEJMp2026393.

77. Creech CB, Walker SC, Samuels RJ. SARS-CoV-2 Vaccines. JAMA. 2021 Apr 6;325(13):1318-1320. doi: 10.1001/ jama.2021.3199. PMID: 33635317.

78. Jiang S, Zhang X, Yang Y, Hotez PJ, Du L. Neutralizing antibodies for the treatment of COVID-19. Nat Biomed Eng. 2020 Dec;4(12):1134-1139. doi: 10.1038/s41551-020-00660-2. 79. Dorans KS, Mills KT, Liu Y, He J. Trends in Prevalence and Control of Hypertension According to the 2017 American College of Cardiology/American Heart Association (ACC/ AHA) Guideline. J Am Heart Assoc. 2018 Jun 1;7(11):e008888. doi: 10.1161/JAHA.118.008888.

80. NCD Risk Factor Collaboration (NCD-RisC). Worldwide trends in body-mass index, underweight, overweight, and obesity from 1975 to 2016: a pooled analysis of 2416 population-based measurement studies in 128.9 million children, adolescents, and adults. Lancet. 2017 Dec 16;390(10113):2627-2642. doi: 10.1016/S0140-6736(17)32129-3.

81. DiBonaventura MD, Meincke H, Le Lay A, Fournier J, Bakker E, Ehrenreich A. Obesity in Mexico: prevalence, comorbidities, associations with patient outcomes, and treatment experiences. Diabetes Metab Syndr Obes. 2017 Dec 22;11:1-10. doi: 10.2147/DMSO.S129247.

82. Vrints CJM, Krychtiuk KA, Van Craenenbroeck EM, Segers VF, Price S, Heidbuchel H. Endothelialitis plays a central role in the pathophysiology of severe COVID-19 and its cardiovascular complications. Acta Cardiol. 2020 Nov 19:1-16. doi: 10.1080/00015385.2020.1846921.

83. Teuwen LA, Geldhof V, Pasut A, Carmeliet P. COVID-19: the vasculature unleashed. Nat Rev Immunol. 2020 Jul;20(7):389-391. doi: 10.1038/s41577-020-0343-0. Erratum in: Nat Rev Immunol. 2020 Jun 4.

84. Evans PC, Rainger GE, Mason JC, Guzik TJ, Osto E, Stamataki Z, et al. Endothelial dysfunction in COVID-19: a position paper of the ESC Working Group for Atherosclerosis and Vascular Biology, and the ESC Council of Basic Cardiovascular Science. Cardiovasc Res. 2020 Dec 1;116(14):2177-2184. doi: 10.1093/cvr/cvaa230.

85. Nägele MP, Haubner B, Tanner FC, Ruschitzka F, Flammer AJ. Endothelial dysfunction in COVID-19: Current findings and therapeutic implications. Atherosclerosis. 2020 Dec;314:58-62. doi: 10.1016/j. atherosclerosis.2020.10.014. 86. The Lancet Public Health. COVID-19-break the cycle of inequality. Lancet Public Health. 2021 Feb;6(2):e82. doi: 10.1016/S2468-2667(21)00011-6. 